\title{
Die SPD, die Meinungsfreiheit und die Konsequenzen der Massenpresse im Kaiserreich
}

\author{
Maria Löblich · Niklas Venema
}

Online publiziert: 1. April 2020

(C) Der/die Autor(en) 2020

Zusammenfassung Mit dem Aufstieg der Massenpresse Ende des 19. Jahrhunderts geriet die SPD in einen Zwiespalt zwischen interner Meinungsfreiheit und dem Umgang mit abweichenden Stimmen. Einerseits konnte sie sich nicht zu Meinungsfreiheit bekennen, ohne diesen Grundsatz auch auf sich selbst anzuwenden, andererseits musste sie stärker als zuvor auf ein geschlossenes Auftreten in der Öffentlichkeit achten. Gestützt auf Medialisierungsforschung und Diskursiven Institutionalismus analysiert der Beitrag die Machtkämpfe innerhalb der Partei, die in öffentlichen Diskursen ausgetragen wurden und in die Presse- und Öffentlichkeitsstrukturen sowie politischen Kontexte im Kaiserreich eingebettet waren. Inhalts- und Dokumentenanalysen zeigen, dass sich die Kritik an mangelnder innerer Meinungsfreiheit in einer medialisierten Umwelt seit den 1890er Jahren verstärkte. Abweichler von der Parteilinie forderten Meinungsfreiheit, um Sichtbarkeit und Legitimation für ihre politischen Ideen und Anerkennung für die Parteiredakteure zu bekommen. Die Organisationsmehrheit hingegen stellte Außenwirkung über Meinungsfreiheit und reagierte damit auf die wachsende Bedeutung öffentlicher Kommunikation.

Schlüsselwörter Kommunikationsgeschichte $\cdot$ Meinungsfreiheit · Geschichte der SPD · Medialisierung · Diskursiver Institutionalismus

Dr. M. Löblich $(\bowtie) \cdot$ N. Venema Institut für Publizistik- und Kommunikationswissenschaft, Freie Universität Berlin, Garystraße 55, 14195 Berlin, Deutschland

E-Mail: maria.loeblich@fu-berlin.de

N. Venema

E-Mail: niklas.venema@fu-berlin.de 


\section{The SPD, freedom of speech and the consequences of mass press in Imperial Germany}

Abstract The study analyzes the consequences of the emerging mass press for the Social Democratic Party of Germany with regard to the value of freedom of speech. It discusses how one of the largest and most successful political parties at that time dealt with the increasing significance of mass media as part of society's functional differentiation in the late 19th century. In view of a more or less differentiated media system, public visibility became a main resource for actors in the German Empire as well as in other Western societies to legitimize their goals and mobilize consent. Earlier than other organizations, political parties realized the importance of public communication for gaining attention, acceptance and legitimation.

However, the emergence of the mass press created a dilemma for the Social Democratic Party in particular. On the one hand, the party that was rooted in the oppressed labor movement demanded freedom of speech from the authoritarian state and therefore had to respect the right to communicate in its own ranks. Freedom of speech was an important value for the Social Democrats especially due to their experience of the oppressive Anti-Socialist-Laws and even ongoing suppression in the Wilhelmine era. On the other hand, the party was increasingly forced to keep a public image of unity in view of the changing media environment. The Social Democrats felt to be persecuted not only by the state but also by liberal, conservative or plain commercial — in the view of the labor movement bourgeois-newspapers. The study analyzes how freedom of speech became a problem for the Social Democratic Party, although the organization strongly identified with this value. The case of the Social Democratic Party is suitable to analyze the question of freedom of speech in view of the changing media environment in the late 19th century, because of the party's organizational structures. The party significantly grew and even had one million members on the eve of World War I. This went along with the establishment of a party bureaucracy and different rules to communicate for officials and ordinary members. Furthermore, several factions of the party fought over power and influence of either revolutionary or reformist ideas.

Against this background, the study analyzes the struggles over power within the party that were fought in public discourses. Therefore, the study builds on previous research on the Social Democratic press that highlighted the complicated relation to the bourgeois press as well as the Social Democratic journalists' attempts to gain autonomy from the party administration. Theoretically, the study relies on the approaches of mediatization and discursive institutionalism. Mediatization depicts societal actors' adaption to a growing importance of media as it can first be detected with the rise of the mass press in the late 19th century. Beyond that, the concept of discursive institutionalism allows describing how actors such as political parties dealt with changes of mediatization via discourses that were embedded in the structures of the press and the public sphere as well as politics in the German Empire.

In order to analyze the internal party discourse about the individual right to communicate for Social Democrats, we conducted qualitative content and document analyses. The analyses are guided by categories derived from the theoretical assumptions. The period of investigation covers the time between the foundation of 
the first unified party of the German labor movement in 1875 and the last party conference before the outbreak of World War I in 1913. The sources comprise minutes of the annual party congresses and by the party steering committee, party journals, brochures and position papers as well as biographical material such as memoirs, (auto-)biographies or letters between party leaders and journalists.

The analyses reveal that criticism of insufficient freedom of speech within the party increased in view of a mediatized environment since the 1890s. With the end of the Anti-Socialist-Laws that had united and radicalized the party members, the individual right to communicate became a recurring issue discussed in different contexts on the annual party conferences until 1913. Party members dissident from the official party line demanded freedom of speech in order to gain visibility as well as legitimation of their political ideas. Apart from that, editors of the party press claimed freedom of speech aiming to find recognition for their work and achieve better equipment of the editorial offices. In contrast, members of the organization's majority put public image before freedom of speech and therefore reacted to the increasing importance of public communication. According to them, public criticism put damage to the party's image. This view was interwoven with the idea of an own party press. Overall, even among critics the distinction between the Social Democratic party press and bourgeois newspapers remained dominant.

Keywords Communication history · Freedom of speech · History of the SPD · Mediatization · Discursive institutionalism

\section{Einleitung}

Das autorisierte und legitimierte Sprechen ist eine Voraussetzung dafür, dass Parteien politische Forderungen artikulieren und Entscheidungen kommunizieren - kurz: ihren Kernaufgaben als intermediären Akteuren gerecht werden können (vgl. Donges und Nitschke 2018, S. 3). Nicht erst durch den jüngsten Medienwandel sind sie mit der Frage konfrontiert, wie sich Wortmeldungen ihrer Mitglieder zu den Regeln verhalten, die sie als politische Organisationen brauchen, um mit einer Stimme sprechen zu können. Schon die vorangegangenen Medialisierungsschübe (vgl. Bösch und Frei 2006, S. 20) dürften Partei(führung)en das Problem bereitet haben, Vielfalt und Kritik im Inneren mit geschlossenem Auftreten nach außen auszutarieren. Die SPD im Kaiserreich liefert ein Fallbeispiel, um dieser Annahme nachzugehen. Dazu veranlassen uns zwei Beobachtungen in Quellen und Literatur.

Erstens zieht sich die Klage, von der „kapitalistischen Presse“ (vgl. Mehring 1908, S. 1) bekämpft zu werden, wie ein roter Faden durch die Parteitage des 19. und frühen 20. Jahrhunderts (vgl. Protokoll 1875; Protokolle 1891, 1907, 1919). Aus Sicht der Sozialdemokraten verkörperte die kommerzielle und zugleich politische Massenpresse den Inbegriff kapitalistischer Interessen (vgl. Requate 2006, S. 128). Die Klage über Verfolgung ging einher mit der allgemeinen Ausbreitung der Presse. Mediale Öffentlichkeit wurde für die SPD wie für andere organisierte Interessen „,zum Objekt politischen Kalküls“ (Stöber 2000, S. 23), hatte für die SPD allerdings spezifische Zugangs- und Gestaltungsbedingungen (vgl. Kunczik 1997; 
Requate 1995, S. 338-346). Obwohl auch ihre eigene Presse bis 1914 stark wuchs, betrachteten die Sozialdemokraten sie im Vergleich zur Massenpresse als „Zwerg“ (Schröder 1907, S. 742). Sie machte 1913 lediglich 2,2\% der Gesamttitelzahl aus (vgl. Groth 1929, S. 468).

Zweitens hatte die Partei, 1875 aus zwei Vorläuferorganisationen entstanden, ${ }^{1}$ Meinungsfreiheit von Anfang an in ihr Programm aufgenommen. Sie war als Forderung an den Staat formuliert, jegliche gesetzliche Einschränkung abzuschaffen (vgl. Protokoll 1875, S. 55). Unter dem Eindruck des Sozialistengesetzes wurde dieser Grundsatz 1891 im neuen Programm noch stärker betont (vgl. Protokoll 1891, S. 5). Auch weil die Diskriminierung der Sozialdemokraten weiter anhielt (vgl. Wehler 2008, S. 1246) und die sozialdemokratische Presse auch nach 1890 strafrechtlich verfolgt wurde (vgl. Danker et al. 2003, S. 49; siehe auch Heine 1901), blieb dieser Grundsatz für die Partei sehr wichtig.

Diese Beobachtungen lassen den Zwiespalt erahnen, in den die SPD im Kaiserreich geraten sein dürfte. Einerseits konnte sie sich nicht zu Meinungsfreiheit bekennen, ohne diesen Grundsatz auch auf sich selbst anzuwenden, andererseits musste sie stärker als zuvor auf ein geschlossenes Auftreten in der Öffentlichkeit achten. Unsere Grundannahme lautet, dass die Ausbreitung der Massenpresse nicht ohne Folgen für den Umgang mit der inneren Meinungsfreiheit bleiben konnte, weil sie es abweichenden Parteimitgliedern erleichterte, sich zu Wort zu melden.

Der vorliegende Beitrag geht dieser Annahme nach. Wir wollen im Einzelnen klären, wie ausgerechnet die SPD, die als demokratische Partei angetreten war, ein Problem mit Meinungsfreiheit in ihren eigenen Reihen bekam. Welchen Stellenwert hatte Meinungsfreiheit im Parteidiskurs, welches Verständnis von Meinungsfreiheit und Parteiöffentlichkeit gab es und wie kam dabei die Presse ins Spiel? Wie ging die Parteiöffentlichkeit mit Kritik an fehlender Meinungsfreiheit um und wie löste die Partei ihr Kommunikationsproblem? Dabei folgen wir dem Medialisierungsansatz (vgl. Bösch und Frei 2006; Donges und Jarren 2014; Meyen 2009), den wir mit dem Diskursiven Institutionalismus (vgl. Carstensen und Schmidt 2016; Schmidt 2008) verbunden haben. Dieser theoretische Rahmen sorgte nicht nur für einen differenzierteren Akteursbegriff als bislang angedeutet, sondern strukturierte die gesamte Analyse des Parteidiskurses im Zeitraum von 1875 bis 1914.

Die SPD ist besonders geeignet, um der skizzierten Diskrepanz nachzugehen, weil sie schon im Kaiserreich Massenpartei war und Organisationsstrukturen entwickelte, die unterschiedliche Kommunikationsregeln für Funktionäre und einfache Mitglieder mit sich brachten. Flügelkämpfe gab es seit der ,scharfen Konkurrenz und (den) Krawallen“ zwischen „Lassalleanern“ und „Eisenachern“ (Faulenbach 2012, S. 14). Die SPD ist andererseits ein spezielles Beispiel, weil ihre Geschichte - bei gewissen Parallelen zur Zentrumspartei - mit Unterdrückung und Ausgrenzung verbunden ist (vgl. Wehler 2008; Große Kracht 2010).

Unsere Studie ordnet sich in die umfangreiche Forschung zur Geschichte der SPD-Presse ein. Sie schließt an Arbeiten zu Strukturen, Lenkung und Wirkung der

\footnotetext{
1 Der von Ferdinand Lassalle gegründete Allgemeine Deutsche Arbeiterverein und die Sozialdemokratische Arbeiterpartei um August Bebel und Wilhelm Liebknecht schlossen sich 1875 zur Sozialistischen Arbeiterpartei Deutschlands (SAPD) zusammen. Ab 1890 trug die Partei den Namen SPD.
} 
Parteipresse an, die auch auf das komplizierte Verhältnis zur bürgerlichen Presse aufmerksam gemacht haben (vgl. Koszyk 1980, S. 4, 1958; Loreck 1977, S. 46-63). Außerdem nimmt sie auf, was Professionalisierungsgeschichten auch erzählt haben: die Frage der ,inneren Autonomie“ der Parteijournalisten (Meißner 2017, S. 135) sowie das „Gerangel um ein Mehr an Meinungsfreiheit“ in den Redaktionen (Sperlich 1983, S. 108-113). Im Unterschied zur parteihistorischen Perspektive, die sich für demokratische Prinzipienfestigkeit interessiert hat (vgl. Plener 1961; Günther 1973; Mittmann 1976; Bloch 2008), wählen wir einen medienwirkungszentrierten Blick auf die SPD. Uns geht es dabei nicht darum, die anhaltende politisierte Debatte fortzuführen, ob die SPD mit ihrer Parteipresse Pluralismus oder einer ,heimlichen Steuerung der Medienöffentlichkeit" Vorschub geleistet hat (Bösch 2004, S. 501; vgl. Boll 2002; Danker et al. 2003; Ressmann 1991; Rudolph et al. 2003). Vielmehr soll dieser Beitrag herausarbeiten, wie diskursive Kommunikationskontrollen vor dem Hintergrund von medialem Wandel und gesellschaftlichen (Macht-)Strukturen zu verstehen sind. Er will Meinungsfreiheit in der Partei als Problem öffentlicher Kommunikation darstellen (vgl. Günther 1973, S. 26).

\section{Meinungsfreiheit, Partei und Presse im Kaiserreich}

In den Zwiespalt zwischen Meinungsfreiheit und Regeln für öffentliche Kommunikation geriet die SPD angesichts politischer, organisatorischer und medialer Veränderungen. Das Reichspressegesetz von 1874 bedeutete eine Liberalisierung der Presse und postulierte Pressefreiheit. Diese wurde aber nicht verfassungsrechtlich garantiert. So war es möglich, dass das Sozialistengesetz 1878 Meinungs- und Pressefreiheit für die Sozialdemokraten außer Kraft setzte. Bis 1890 blieben ihnen Organisationen, Versammlungen und Publikationen verboten (vgl. Stöber 2014, S. 144-146; Wetzel 1975, S. 185-202). Bis zum Ersten Weltkrieg sammelte die SPD nahezu 1,1 Mio. Mitglieder (vgl. Fricke 1987, S. 308). Ab 1890 konnte sie bei den Reichstagswahlen große Erfolge verbuchen und 1912 34,8\% der Stimmen erreichen (vgl. Steinbach 1990, S. 5-6). Die Organisation der Partei war auch nach Ende des Sozialistengesetzes erschwert. Erst um die Jahrhundertwende wich das System der Vertrauenspersonen zunehmend einer zentralistischen Organisation. Oberste Einrichtung war der jährliche Parteitag, auf dem die Delegierten der Wahlkreise über die Parteipolitik und die Zusammensetzung der Parteileitung bestimmten (vgl. Ritter 1963, S. 46-58). Mit der wachsenden Partizipation im Staat entwickelte sich ein Apparat hauptamtlicher Funktionäre, zu dem auch die Redakteure der Parteizeitungen gehörten (vgl. Faulenbach 2012, S. 24-25; Koszyk 1989, S. 180). Die Vorstände bestimmten die Schwerpunkte der Parteitage und legten gemäß Organisationsstatut eine vorläufige Tagesordnung fest. Die Mitglieder konnten weitere Themen beantragen (vgl. Schröder 1910, S. 359-338).

Neben den Parteitagen waren die Reflexionsorgane Die Neue Zeit und Sozialistische Monatshefte wichtige Foren für die Diskussion der Parteigrundsätze. Der revolutionäre Marxismus, unter dem Eindruck des Sozialistengesetzes als offizielle Programmatik etabliert, geriet ab den 1890er Jahren in ein Spannungsverhältnis zur sozialreformerischen Praxis der Partei. Der Parteitheoretiker Eduard Bernstein 
forderte die Doktrin mit seinen Thesen zur Anpassung der marxistischen Theorie (,Revisionismus“) heraus. Reformer wie Georg von Vollmar strebten eine Verbesserung der Verhältnisse im bestehenden System an (vgl. Wehler 2008, S. 1046-1047). Vor Ausbruch des Ersten Weltkrieges konnte sich der rechte Flügel in der Partei nicht durchsetzen (vgl. Faulenbach 2012, S. 27-31).

Zwischen 1875 und 1878 war es zu einer ersten Gründungswelle lokaler sozialdemokratischer Zeitungen gekommen. Das Sozialistengesetz verbot die Publikationen weitgehend. Trotzdem verlegten Sozialdemokraten auch in dieser Zeit kurzlebige, vermeintlich unpolitische Blätter (vgl. Koszyk 1980, S. 14; Sperlich 1983, S. 120). Ab 1890 folgte eine zweite Gründungswelle bis zum Ausbruch des Ersten Weltkriegs (vgl. Boll 2002, S. 33-42). Pressekommissionen kümmerten sich um Linientreue sowie Anstellung und Entlassung von Redakteuren (vgl. Sperlich 1983, S. 39-42). Trotz ihres Wachstums konnte die sozialdemokratische Presse mit der Massenpresse nicht konkurrieren. Diese Konkurrenz lieferte fortwährend Diskussionsstoff, auch wenn für die eigenen Blätter moralische Überlegenheit beansprucht wurde (vgl. Danker et al. 2003, S. 43-45).

\section{Medialisierung und Diskursiver Institutionalismus}

„Medialisierung“ steht für die Reaktionen eines gesellschaftlichen Akteurs, die sich auf „den Strukturwandel des Mediensystems“ (Donges 2008, S. 154) beziehen oder auf den ,generellen Bedeutungszuwachs medial vermittelter öffentlicher Kommunikation“ (Meyen 2009, S. 3). Wir haben an Studien zur Medialisierung von Organisationen angeknüpft, die diese Reaktionen mit anderen gesellschaftlichen Entwicklungen verflochten sehen (vgl. Meyen 2009, S. 3). Politiker benötigen mediale Aufmerksamkeit und öffentliche Legitimation, um Zustimmung, Wählerstimmen, Macht zu erhalten (vgl. Meyen 2009, S. 3; Meyen et al. 2014). Diese Phänomene sind bereits im Kaiserreich zu finden (vgl. Kohlrausch 2005; Geppert 2007). Wahrnehmungen der medialen Umwelt gelten neben Strukturen und Kommunikation als Indikator für die Medialisierung einer Organisation (vgl. Donges 2008, S. 149-156; Donges und Jarren 2014, S. 190). Dieses Nebeneinander von Indikatoren hat unserem Erkenntnisinteresse allerdings nicht weitergeholfen. Wir wollten verstehen, wie aus Wahrnehmungen und Deutungen Definitionshoheiten und Regeln des Sprechens in einer (veränderten) Medienumgebung werden, die dann Veränderungen von Organisationsstrukturen und Kommunikation anleiten. Der Diskursive Institutionalismus lieferte diese Verknüpfung mithilfe von Ideen und Diskurs. Diese Konzepte haben die Historiker Frank Bösch und Norbert Frei (2006, S. 8-9) auch für historische Medialisierungsforschung empfohlen, denn sie machen den Zusammenhang zwischen Medienentwicklung, Gesellschaftsgeschichte und sozialer Selbstdeutung deutlich. Auch der hier benutzte kollektive Akteursbegriff, der das differenzierte und konflikthaltige Innenleben sowie die porösen Grenzen von Organisationen betont (vgl. Donges und Nitschke 2018, S. 5), legte die Integration des diskursiven Institutionalismus nahe. Die Medialisierungsforschung lieferte uns die Grundannahmen zur gesellschaftlichen Wirkung von Medien. Der Diskursive Institutionalismus stellte die begrifflichen Werkzeuge bereit, um die diskursive Auseinandersetzung in der 
Partei zu untersuchen. Er klärte, welche Ideen zur Funktions- und Wirkungsweise von Presse und Öffentlichkeit aufgrund welcher Umstände in der Partei artikuliert, durchgesetzt und reproduziert wurden und wie diese Ideen handlungsleitend wurden.

Die Politikwissenschaftlerin Vivien A. Schmidt (2008) interessiert sich für die Entwicklung und Veränderung von politischen Ideen und Institutionen, die über Diskurse in und zwischen Organisationen untersucht werden können. Diese Diskurse sind mit Macht verbunden. In Diskursen werden (normative und kognitive) Ideen bekämpft, erhalten oder durchgesetzt. Akteure beziehen sich bei der Interpretation politischer Probleme und der Legitimation ihrer Vorschläge auf Ideenstrukturen, die den anderen zur Gewissheit geworden sind (ideational structures). Ideenstrukturen ermöglichen und beschränken zugleich Denken und Sprechen (power in ideas). Akteure reproduzieren Ideenstrukturen, sind aber auch in der Lage, sich kritisch mit diesen auseinanderzusetzen und sie zu verändern. Akteure versuchen, andere $\mathrm{zu}$ überreden, abzustrafen oder zu unterstützen (power through ideas). Sie sind in unterschiedlichem Maße mit struktureller Macht ausgestattet, was ihnen mehr oder weniger Einfluss auf die Bedingungen des Diskurses ermöglicht (power over ideas; vgl. Carstensen und Schmidt 2016, S. 305, 320-324).

Institutionelle Kontexte beeinflussen den Verlauf von Diskursen. Damit sind strukturelle Faktoren aus Geschichte und Gegenwart gemeint, die verinnerlicht wurden, sich zu Ideenstrukturen verfestigt haben und deshalb mit Macht verbunden sind. Sie formen Identität, Selbstverständnis und Interessen eines Akteurs (vgl. Carstensen und Schmidt 2016, S. 329). Sie bilden Gewissheiten ,im Hintergrund“ des Bewusstseins und sorgen für Kontinuität im Denken und Handeln. Die background ideational abilities einer Organisation sorgen dafür, dass Akteure solche Gewissheiten entwickeln und aufrechterhalten, etwa Weltanschauungen oder Überzeugungen zur Funktionsweise von Massenmedien (vgl. Schmidt 2008, S. 314; Carstensen und Schmidt 2016, S. 329). Um den Wandel von Ideen zu erklären, führt Schmidt den Begriff der foreground discursive abilities ein. Sie befähigen Akteure, auch außerhalb der institutionellen Kontexte zu denken und zu sprechen, in denen sie sich bewegen. Sie stoßen dabei auf andere, die etablierte Ideen verteidigen und erhalten wollen (vgl. Schmidt 2008, S. 314).

Die Verbindung von Medialisierung und diskursivem Institutionalismus erschloss die Sozialdemokratie im Kaiserreich als in zeitlich bedingte Presse- und Öffentlichkeitsstrukturen sowie politische Kontexte eingebettete und in sich ausdifferenzierte Organisation, in der Machtkämpfe in Diskursen ausgetragen wurden. Die Mitglieder waren mit ihrem spezifischen Zugang zur (Partei-)Öffentlichkeit zu untersuchen sowie mit ihren - je nach Parteianbindung oder Herkunfsmilieus unterschiedlichen - Interessen an und Ideen zu Meinungsfreiheit, Presse und Öffentlichkeit.

\section{Untersuchungskategorien und Quellen}

Ein theoretisch abgeleitetes Kategoriensystem leitete die Inhalts- und Dokumentenanalyse an. Wir erfassten den Diskurs zu Meinungsfreiheit mit folgenden Kategorien: Verständnis von Meinungsfreiheit, Presse und öffentlicher Kommunikation, von Sozialismus (Schmidt zufolge: normative und kognitive Ideen unterschiedlichen Ab- 
straktionsgrads), diskursive Praktiken (power through ideas). Um zu erklären, warum Akteure bestimmte Ideen vertreten, haben wir außerdem institutionelle Kontexte einbezogen. Die Kategorien waren hier: Ideenstrukturen, Foren sowie nicht-diskursive Strukturen (Ebenen: Biographie, Partei, Pressesystem, Politisches System, Recht). Die Ausdifferenzierung von institutionellen Kontexten auf verschiedenen Analyseebenen legt Schmidt nahe. Sie verweist auf das Habituskonzept Bourdieus, auf organisationsinterne Rollen und politische Systemstrukturen (vgl. Schmidt 2008).

Der Untersuchungszeitraum erstreckte sich von der Gründung der SAPD bis 1913, dem letzten Parteitag vor dem Ersten Weltkrieg. Er deckt Expansion und Differenzierung der Presse bis zur Krise nach Kriegsausbruch ab und ermöglichte es, den parteiöffentlichen Diskurs als Reaktion auf den Medienwandel zu analysieren. Kriterien der Quellenauswahl waren Nähe zum Parteidiskurs und Einflusspotential auf die Partei. Protokolle der Parteitage der SAPD/SPD wurden als wichtigste parteiöffentliche Diskussionsforen herangezogen. Sie dokumentieren den Diskurs für die Zeit vor 1890 allerdings deutlich weniger ausführlich als für die Zeit danach. Quellen waren darüber hinaus Die Neue Zeit (ab 1883), Sozialistische Monatshefte (ab 1897), Artikel im Zentralorgan Vorwärts sowie Broschüren und Denkschriften, die Parteitagsdiskussionen flankierten oder vor- und nachbereiteten. Aufschluss über informelle Prozesse geben diese Quellen nicht. Zudem zeigen sie lediglich die Wahrnehmung der Partei, nicht aber die tatsächliche Berichterstattung bürgerlicher Zeitungen über die SPD. Weitere Quellen sind Memoiren und Biographien zur Einordnung der Akteure, Parteiausschussprotokolle (ab 1912) und Briefwechsel, die Anhaltspunkte für den informellen Austausch in der Parteiführung gaben.

Wir haben im Material zunächst festgestellt, wann und zu welchen Anlässen Meinungsfreiheit gefordert wurde. Anschließend interessierte uns, wie sich der Streit intensiviert hat (Häufigkeit und Länge der Beschäftigung auf Parteitagen, in den Zeitschriften, Parteikontext der Akteure). Wir haben diese Erkenntnisse auf die Entwicklung der Massenpresse bezogen. Nach diesem Analyseschritt haben wir über Zeit und Anlässe hinweg Muster der Bezugnahme auf Meinungsfreiheit rekonstruiert, die anschließend wieder mit Blick auf Akteure, Anlässe und Parteientwicklung $\mathrm{zu}$ kontextualisieren waren.

\section{Sichtbarkeitskämpfe zwischen Abweichlern und Mehrheit}

Wir entfalten in vier Schritten unsere Erkenntnis, der zufolge die Ausbreitung der Massenpresse die Hörbarkeit abweichender Stimmen förderte und die Partei in einem Zwiespalt gefangen hielt, was ihren Umgang mit innerer Meinungsfreiheit anging. Im ersten Schritt zeigen wir, wie sich ab 1890 die Kritik an innerer Meinungsfreiheit intensivierte und welche Ereignisse dazu Anlass boten. Dann betrachten wir die Abweichler, die Meinungsfreiheit aus eigenen Professionalisierungsinteressen einforderten und das etablierte Denken der moralisch überlegenen und disziplinierten Parteipresse und -öffentlichkeit infrage stellten (foreground discursive abilities). Drittens rücken die Bewahrer dieser Ideenstruktur aus dem Parteizentrum in den Blick, die diesen Forderungen eine Absage erteilen mussten (background ideational abilities). Hier wird auch erläutert, wie sich die Bewahrer mit ihrer innerparteilichen 
Macht über Ideen (power over ideas) durchsetzen konnten, warum die „Einhegung“ der Kritiker aber nicht ohne die gesellschaftlichen Machtkonstellationen zu verstehen ist und dies ambivalente Folgen für die Partei hatte. Thesen (Kursivsatz) gliedern unsere Argumentation.

\subsection{Verstärkung der Kritik ab 1890}

\section{Mit dem Eintritt in die medial entfesselte Gesellschaft nach 1890 wird Mei- nungsfreiheit innerhalb der Sozialdemokratie zum Problem.}

Mit den gewachsenen Mitgliederzahlen sowie der wieder aufgelebten politischen Arbeit nach Ende des Sozialistengesetzes verstärkten sich die „Fliehkräfte im Inneren“ (Schmidt 2013, S. 176). Die Vielfalt der Meinungen nahm zu und konnte über einen dichteren Medienverbund als zuvor weite Verbreitung finden. $\mathrm{Zu}$ diesem gehörten neue parteieigene Angebote. Diffamierungen durch die Presse waren aus Sicht der Genossen weiterhin die Regel. Schon der erste Parteitag nach Ende des Sozialistengesetzes machte den Zwiespalt deutlich, mit dem die SPD nun besonders dringlich umgehen musste: das Austarieren der nötigen Geschlossenheit nach außen mit Meinungsfreiheit im Inneren. Wilhelm Liebknecht kam gleich zu Beginn seiner Eröffnungsrede auf den Druck der Berichterstattung zu sprechen: „Es ist Ihnen bekannt, daß in den letzten Tagen durch die gegnerische Presse verbreitet worden ist, die Sozialdemokraten hätten zwar im ersten Moment in großmüthiger Aufwallung erklärt, der Eintritt zum Kongreß solle frei, seine Verhandlungen öffentlich sein, aber (...) der Beschluß sei zurückgenommen worden, weil wir (...) viel schmutzige Wäsche zu waschen hätten. Wohlan, nun tagen wir hier im Lichte der vollsten Oeffentlichkeit; die Vertreter der Presse aller Parteien dürfen anwesend sein." (Protokoll 1890, S. 12). Dieses Öffentlichkeitsverständnis - die Parteipresse als Waffe gegen die feindliche bürgerliche Presse - erforderte Geschlossenheit der parteieigenen Kommunikation und letztlich Kontrolle, zumal die eigene Presse weiter strafrechtlich verfolgt wurde (vgl. Danker et al. 2003, S. 49). So sah der von Ignaz Auer präsentierte Entwurf für ein Organisationsstatut vor, „daß der Parteivorstand auch die prinzipielle Haltung der Parteiorgane zu kontrollieren hat" (Protokoll 1890, S. 124). Der Entwurf wurde beschlossen, jedoch machten sich manche Delegierte Sorgen. So zollte etwa August Keßler aus Bernburg durchaus dem Prinzip der Kontrolle Tribut, forderte aber angesichts der „Auslegung Auers“, wonach einzelne Artikel zu prüfen wären, „die Preßfreiheit zu sichern“ (Protokoll 1890, S. 140). Ein Jahr später nahm Wilhelm Liebknecht die „Opposition der Jungen“ zum Anlass, den Genossen einzuschärfen, Parteikritik intern zu äußern. „Aber wir sind eine organisierte, geschlossene Partei, und es ist notwendig, daß man diejenigen Instanzen durchgeht, die man durchgehen muß, bevor man an die Oeffentlichkeit mit Beschwerden und Anschuldigungen geht.“ (Protokoll 1891, S. 129) August Bebel beklagte, ,(d)aß man sich alle die Erfahrungen und öffentlichen Erörterungen, die wir ja als demokratische Partei zu machen gezwungen sind, ad notam nimmt und bei gelegener Zeit benutzt“" (Protokoll 1897, S. 98). Er wiederholte auch später Liebknechts Ermahnung (vgl. Protokoll 1902, S. 125). 
Auch dass die Beschäftigung mit Meinungsfreiheit immer wiederkehrte, umfangreicher und medial verdichtet wurde, spricht dafür, dass die Partei hier keine Ruhe mehr fand. Mehrere Parteitage setzten die Diskussion über die Mitarbeit in der bürgerlichen Presse ausführlicher als zuvor fort (1891, 1901, 1903). Der Parteitagsbeschluss von 1903 untersagte, dass „Parteigenossen (...) an bürgerlichen Preßunternehmungen tätig sind, in denen an der sozialdemokratischen Partei gehässige oder hämische Kritik geübt wird“. Er ging auf einen Vorschlag des Vorstands zurück. Für Vertrauensämter sollten diese Genossen nicht infrage kommen (vgl. Protokoll 1903, S. 117-118; Parteivorstand 1903a, S. 3). Auch parteinahe, unabhängige Medienangebote, Versammlungen und sogar einzelne Vorträge wurden zum Thema gemacht. Dazu zählten die Sozialistischen Monatshefte (Parteitage 1895, 1901, 1902, 1909; Parteiausschuss 1913), die Zusammenkünfte und ein Flugblatt der „Berliner Opposition“ (Parteitage 1890, 1891), Schriften und ein Vortrag von Eduard Bernstein (Parteitage 1899, 1901) sowie der Vorwärts (Parteitag 1905). Die „Opposition der Jungen“, denen die Reichstagsfraktion zu zahm geworden war (vgl. Wienand 1976), nahm nahezu den gesamten ersten Verhandlungstag 1890 ein und zog sich ein Jahr später sogar über drei von sechs Tagen hin. 1901 verbrauchte der Kongress ebenfalls die Hälfte der Zeit, um über Eduard Bernstein zu diskutieren. Es ging um mehrere Fragen: Ob der gerade aus dem Londoner Exil zurückgekehrte Sozialdemokrat einen Vortrag über ,wissenschaftlichen Sozialismus“ im Berliner Sozialwissenschaftlichen Studentenverein hätte halten dürfen (vgl. Bernstein 1991, S. 225; Protokoll 1901, S. 138-139.), wie die ,bürgerliche Presse seinen Vortrag fruktifizirte“, wie Bebel betonte (Protokoll 1901, S. 167), ob nicht Bernstein dem Presselob hätte entgegentreten müssen und was die Parteipresse versäumt hatte (Protokoll 1901, S. 132-190). In dieser Debatte rückten auch die Sozialistischen Monatshefte in die Kritik. Ihre Autoren sahen sich dem Vorwurf ausgesetzt, gegnerischen Zeitungen zuzuspielen und der Partei zu schaden (vgl. Protokoll 1901, S. 170-172). Die Auseinandersetzung um diese Zeitschrift, der die Anerkennung als Parteiorgan verweigert wurde, war eng verbunden mit der Kritik am ,wissenschaftlichen Zentralorgan“ Die Neue Zeit (vgl. Stampfer 1957, S. 54), die auf dem Parteitag 1901 und noch deutlicher 1902 artikuliert wurde. Reformer und Revisionisten wie Georg von Vollmar und Eduard David beschwerten sich über Karl Kautskys Redaktionspolitik, die streng der marxistischen Parteilinie folgte, und wünschten Anerkennung der Monatshefte. Auch mit dem Thema der Wirksamkeit der Parteipresse ergab sich eine neue Gelegenheit, Meinungsfreiheit anzusprechen (etwa 1893, 1895, 1896, 1901, 1902, 1909, 1912, 1913). 1905 gerieten Parteiredakteure des Vorwärts in Konflikt mit Vorstand und Berliner Pressekommission. Hinter der abweichenden Haltung, die der Chefredakteur Kurt Eisner und seine Mitstreiter zu Massenstreiks bezogen hatten, stand die Frage, wie das Zentralorgan bei parteiinternen Differenzen berichten sollte. Durch die Doppelfunktion als Berliner Lokal- und Zentralorgan war dies erschwert (vgl. Grau 2001, S. 210-219).

Meinungsfreiheit wurde nach 1890 auch deshalb zum Problem, weil sich die diskursive Praxis änderte. Schon zuvor war Widerspruch laut geworden worden, sobald Beziehungen zur bürgerlichen Presse oder Neugründungen angeprangert wurden. Das geschah aber entweder nicht mit dem Argument „Meinungsfreiheit“, wie im Fall der linksliberalen Frankfurter Zeitung und ihres Verlegers Leopold Sonnemann 
(vgl. Protokoll 1876, S. 44-54). Oder das Argument wurde nicht offensiv für eine Kritik an der Parteiführung eingesetzt, etwa bei dem Parteiausschluss des Parteiredakteurs und Reichstagsmitglieds Wilhelm Hasselmann und des Exilpresse-Herausgebers Johann Most, beide Anhänger des Anarchismus (vgl. Protokoll 1880, S. 25; Wienand 1976). Meinungsfreiheit wurde informell angemahnt, wie in Briefen von Friedrich Engels an Parteiführer (vgl. Loreck 1977, S. 74; Sperlich 1983, S. 226). Freigesetzt in eine verdichtete Medienwelt, problematisierte nun eine gewachsene Gruppe von Kritikern die Regeln für öffentliche Kommunikation und übte offene Kritik an der Parteiführung. Allerdings schränkt die Quellenlage diesen Befund ein. In der Zeit des Sozialistengesetzes fanden nur unregelmäßig Parteikonferenzen statt, deren Diskussionen zudem nicht wörtlich, sondern zusammengefasst wiedergegeben wurden.

$\mathrm{Zu}$ den Kritikern gehörten Vertreter von Minderheiten: anfangs Linksoppositionelle, später vor allem der rechte Flügel sowie Parteijournalisten. Sie konnten sich leichter als zuvor zu Wort melden, zumindest in den Monatsheften sowie in Broschüren und Pamphleten (vgl. etwa Braun 1903b; Büttner et al. 1905; Calwer 1894; Vollmar 1892, 1903). Letztere waren ab 1890 schneller, billiger und in höheren Auflagen herstellbar (vgl. Kohlrausch 2005, S. 56-57).

\subsection{Das Problem der inneren Meinungsfreiheit}

Die Herausforderer kritisierten Unterdrückung und Bevormundung. Sie wiesen auf die Chancen und Funktionsweisen der modernen Presse hin und stellten damit die Autorität des SPD-Presseverständnisses infrage.

Die Kritik stützte sich auf zwei zentrale Argumente, die über die genannten Themen und die Zeit zwischen 1890 und 1914 hinweg wiederkehrten: demokratischer Wesenskern und Erfolg der Parteipresse. Das Demokratie-Argument tauchte auch in den Nachlesen zur Reichstagswahl von 1903 auf, aus der die Partei stärker denn je hervorgegangen war (vgl. Wehler 2008, S. 1046). Der Reichstagsabgeordnete Vollmar (1903, S. 18-19) beschwerte sich über den „herrische(n) Ton des Schulmeisters“ Bebel, der Bernstein in der Neuen Zeit kritisiert hatte (vgl. Bebel 1903). Kautsky habe Bebels Beitrag in ,den auffälligen Formen eines amtlichen Erlasses“ veröffentlicht, obwohl es sich um „Meinungsäußerungen (...) eines gleichberechtigten Mitarbeiters“ handele. Er begründete das „Recht (...) jedes Parteigenossen auf freie Meinungsäußerung“ damit, dass die SPD ,eine demokratische und keine autoritäre Partei sein" wolle (Vollmar 1903, S. 18). Auch der Abgeordnete Wolfgang Heine (1903, S. 477-478) hatte den Wahlerfolg genutzt und „Bevormundungsversuche(n)“ und „unbrüderliche(n) Zwang“ in der Partei thematisiert. Heine wurde im selben Jahr wegen seiner Mitarbeit in bürgerlichen Zeitungen angegriffen. Im VorwärtsKonflikt argumentierten Redakteure damit, dass in einer „Partei der Oeffentlichkeit“ eine „ehrliche Kritik von Mißständen“ erlaubt sein muss (Büttner et al. 1905, S. 139). Auf über 140 Seiten veröffentlichten sie die „Aktenstücke“ zum Konflikt.

Das zweite Argument griff die Unzufriedenheit mit der Parteipresse auf. Es knüpfte "Wirksamkeit“ an Freiräume für Journalisten und verbesserte Ausstattung (vgl. Protokoll 1896, S. 67-80, 1901, S. 133-138, 1913, S. 8; Schröder 1907, S. 742). 
Der Redakteur der Wiener Arbeiter-Zeitung Karl Leuthner (1910, S. 492, 494) war der Ansicht, dass eine Zeitung erst dann ,die öffentliche Meinung mit fortreißen“ kann, wenn Journalisten aus „Einengungen“ befreit sind. Eine Redaktion sei ,nicht im stande fruchtbringend zu wirken“, wenn sie sich „bis zu einem gewissen Grade nicht frei bewegen kann“, warnte auch Richard Calwer in den Monatsheften (1901, S. 704). Der freie Journalist, der ein paar Jahre Redakteur des Braunschweiger Volksfreunds gewesen und dann mit kolonialistischen Tönen aufgefallen war (vgl. Bloch 2008, S. 15), musste sich auf dem Parteitag rechtfertigen (vgl. Protokoll 1901, S. 197). Calwer (1906) erneuerte seine Kritik an „Disziplin und Meinungsfreiheit“ in der Partei, als klar wurde, dass seine Partei-Korrespondenz Wirtschaftliche Wochenschau gescheitert war (Th. 1906). Schon 1893 hatte Heinrich Braun die Parteileitung kritisiert, nicht nur die „Geringschätzung“ und „stiefmütterlich(e)“ Ausstattung der Parteipresse, sondern auch die fehlende „Gesinnung größter kritischer Freiheit“ (Braun 1893, S. 515, 517, 519). Mit seinem Archiv für soziale Gesetzgebung und Statistik war es ihm noch unter dem Sozialistengesetz gelungen, sich einen eigenen Kanal zu schaffen, der in sozialliberale wissenschaftliche Kreise hineinreichte (vgl. Braun-Vogelstein 1932, S. 98-114). Die Leitung der von ihm mitgegründeten Neuen Zeit hatte er nach ,gereizten Auseinandersetzungen“ verlassen: Braun habe ,auf Akademiker und Liberale wirken“" wollen, er selbst ,auf intelligente Proletarier“", so Kautsky (1960, S. 541-542). Ein späteres Zeitschriftenprojekt scheiterte am Geld, aber auch am Streit um die Mitarbeit an bürgerlichen Zeitungen. In dessen Mittelpunkt war Braun geraten, als er seine dritte Ehefrau Lily von Gisycki verteidigen wollte, die wie Paul Göhre oder Georg Bernhard in Maximilian Hardens Zukunft geschrieben hatte (vgl. Braun 1903a, 1903b; Protokoll 1903).

Die Abweichler betonten die Vorteile von breiter medialer Öffentlichkeit und wiesen auf das Spektrum und die Funktionsweise bürgerlicher Presseangebote hin. Damit stellten sie die Autorität des SPD-Presseverständnisses infrage. Im Streit um Bernsteins Vortrag relativierte Wolfgang Heine die Vorwürfe: Es mache einen Unterschied, ob ,ein ganz untergeordnetes Organ“ wie Welt am Montag über die Partei berichte oder das „Weltblatt“ Frankfurter Zeitung (Protokoll 1901, S. 146). Heine schrieb selbst für das Berliner Tageblatt und die Vossische Zeitung, was auch 1913 noch den Parteiausschuss beschäftigte (vgl. SPD 1980, S. 57; Sperlich 1983, S. 183). Georg Gradnauer, Reichstagsabgeordneter und Vorwärts-Redakteur, argumentierte in derselben Debatte mit dem Wissen um die Logik der Presse. Bürgerliche Blätter lebten ja davon, ,in unseren Reihen Wirrniß anstiften zu wollen“. Er fragte, ob sich die Partei ,fortwährend nervös aufregen lassen“ wolle ,durch die Spaltungsfabeleien einer gegnerischen Presse“ (Protokoll 1901, S. 135). Heinrich Braun wies später darauf hin, dass die bürgerliche Presse ,unzählige der verschiedenartigsten Nuancen auch in ihrer Stellung zur Socialdemokratie“ aufwies (Braun 1903a, S. 3). Der Bruder des Parteiredakteurs Adolf Braun hatte sich im Vorfeld des Kongresses im Vorwärts einen Schlagabtausch mit dem Parteivorstand geliefert. Unter der Überschrift „Freiheit der Meinungsäußerung und Parteivorstand“ warnte er dabei vor einer „chinesischen Mauer“ und betonte, dass es das ,unabweisbare Interesse“ der Partei sei, sich auch zukünftig bürgerlicher Zeitungen zu bedienen (Braun 1903a, S. 3; vgl. Parteivorstand 1903a, 1903b). Schon zehn Jahre zuvor war das moralische Öffentlichkeitsverständnis von ihm infrage gestellt worden (vgl. Braun 1893, 
S. 520). Das taten auch Eduard Bernstein (1909) und Karl Leuthner (1910) in den Monatsheften. Ein sozialdemokratischer Artikel könne in einem nichtsozialdemokratischen Blatt „mehr am Platz sein“, so Bernstein (1909, S. 1094), ,nämlich sobald es sich um die Einwirkung auf ein grösseres oder anders geartetes Publikum handelt". Bernstein hatte im Londoner Exil Presse und Publikum im „Sensationsprozess“ über Oscar Wilde beobachtet und einen ,englischen Agitator“" studiert (Bernstein 1895, S. 175, 1896, S. 11).

Auch die Vorschläge der Herausforderer stellten die Ideenstruktur der sozialdemokratischen Presse infrage: Mitarbeit in bürgerlichen Zeitungen, Reform - jedoch nicht Abschaffung - der Pressekommissionen, Anerkennung und bessere Bezahlung von Berufsjournalisten, Orientierung an der Massenpresse (vgl. Calwer 1894, 1901, 1906; Stampfer 1903; Schröder 1910; Bernstein 1909; Leuthner 1910). De facto hatte sich die Parteipresse längst für bestimmte Organisations- und Funktionsweisen der Massenpresse geöffnet, nur spiegelte die offizielle Linie das kaum wider (vgl. Löblich und Venema 2018; Sperlich 1983, S. 149). Alex Hall (1977, S. 192-193) hat Sensations- und Skandalberichterstattung in der Parteipresse der 1890er Jahre gefunden.

\section{Berufskontexte und Einkommensquellen verweisen auf unterschiedliche Inte- ressen und Spielräume bei der Problematisierung von Meinungsfreiheit.}

$\mathrm{Zu}$ den Akteuren mit foreground discursive abilities gehörte entweder ein bürgerliches Elternhaus, das das Studium bezahlte, oder zumindest ein Handwerkerdasein, das Zeit für autodidaktische Bildung ließ (vgl. Wienand 1976, S. 219). Diese Akteure verfügten über journalistische Erfahrung oder besaßen Gespür für öffentliche Resonanz, wie der ehemalige Pfarrer Paul Göhre, der eine aufsehenerregende Sozialreportage sowie eine weit verbreitete Rede über seinen Weg in die Sozialdemokratie verfasst hatte (vgl. Göhre 1891; Protokoll 1903, S. 236; Bloch 2008). Einige waren Mitglieder im Reichstag.

Sortiert man die Akteure nach der Erwerbsquelle, dann werden unterschiedliche diskursive Spielräume und Interessen sichtbar. Manche waren nicht (mehr) auf Einkünfte aus der Partei angewiesen. Georg von Vollmar, Eduard David und Paul Göhre hatten wohlhabende Frauen geheiratet (vgl. Kampffmeyer 1930, S. 72-73, 86-89; Matthias und Miller 1966, S. XV; Brenning 1980, S. 234). Sie gehörten wie Wolfgang Heine, der sein Geld als Rechtsanwalt verdiente, zu dem ,gut angezogenen" Kreis, der sich in Heinrich Brauns Berliner Villa traf (vgl. Stampfer 1957, S. 85-87). Dem vernetzten Österreicher Braun gelang es außerhalb der Partei, Geld für seine Projekte aufzutreiben (vgl. Braun-Vogelstein 1932; Kautsky 1960, S. 521, 533). Dieser materiell unabhängigen Gruppe dürfte es leichter gefallen sein, Parteiführern Willkür und Autoritarismus vorzuwerfen, als einer anderen, die von der Partei bezahlt wurde. Eduard David unterstellte Kautsky „Mundtodtmachungen“, sprach von ,fruchtlosen Beschwerdeinstanzen“ und ,niedermachen“ (Protokoll 1902, S. 127). Eduard Bernstein und er selbst dienten als Beispiel. Vollmar beschwerte sich über Kautskys „Geist der Einseitigkeit, (...) der Unduldsamkeit““ (Protokoll 1902, S. 139-140). Einige Wortführer der 1891 ausgeschlossenen Berliner „Opposition der Jungen“ markieren die Ausnahme dieser diskursiven Praxis (power through ideas), etwa Wilhelm Werner, dessen kleines Druckergeschäft ,,auf Arbei- 
terkundschaft berechnet(e)“" war und die in Parteibesitz befindliche Berliner VolksTribüne druckte (Wienand 1976, S. 237; vgl. Bezirksleitung 1987, S. 326-327). Werner regte sich auf, dass ,Jemand, der eine andere Meinung hat, mit Schlagworten wie Anarchist, Polizeispitzel und unberechtigte Opposition einfach beseitigt wird“. Er zielte damit auch auf die Reichstagsabgeordneten Bebel und Grillenberger (vgl. Protokoll 1890, S. 70, 43-45; Parteivorstand 1891).

Kritiker, die Parteiredakteure waren oder von der Partei abhängige freie Journalisten, waren zurückhaltender und behielten die Abgrenzung zur bürgerlichen Presse bei (vgl. etwa Calwer 1901, S. 706-707; Büttner et al. 1905, S. 98). Überhaupt suchte die Mehrheit der Parteijournalisten in Sachen Meinungsfreiheit nicht die große Parteiöffentlichkeit (vgl. Meißner 2017). Die Resolution gegen die Mitarbeit in der bürgerlichen Presse trug sie mit, so auch Georg Gradnauer (vgl. Protokoll 1903, S. 263-264). Dieser hatte sich aber zuvor dagegen gewehrt, im Vorwärts per se abweichende Meinungen anzuprangern und ihn ,so (zu) redigieren, daß er stets und immer gerade Bebel's Anschauungen entspricht“ (Protokoll 1901, S. 149). Der Vorwärts-Redakteur hatte seine Kritik weich verpackt. Es sei ihm ,nicht angenehm, gegen einen Führer und Meister wie Bebel auftreten zu müssen“ (Protokoll 1901, S. 133). Friedrich Stampfer (1903) meldete „Gewissensfragen“ an, als er dem Vorstand in Sachen Mitarbeit in bürgerlichen Zeitungen widersprach. Er hatte gerade seine Anstellung bei der Leipziger Volkszeitung verloren und musste sich als „Hungerleider“ durchschlagen (Stampfer 1957, S. 72). Auch Eduard Bernstein gehört nach 1900 zu den verhaltenen Kritikern, wohl aus Dankbarkeit, weil Parteigenossen die Aufhebung seines Haftbefehls erwirkt hatten und weil er „ohne Vermögen“ zurückgekehrt mit „literarische(m) Erwerb“ wieder Boden unter den Füßen gewann (Bernstein 1991, S. 224). Als Reichstagsabgeordneter ab 1903 habe er ein ,eher zurückgezogenes Parteidasein“"geführt (Morina 2017, S. 435).

Wir haben unter den Parteikritikern zwei Interessen an dem Diskurs um innere Meinungsfreiheit identifiziert, die Folgen des Medialisierungsschubs gewesen sein dürften. Erstens ging es um Sichtbarkeit für abweichende politische Haltungen: Sichtbarkeit, um den politischen Kurs zu ändern und das Politikerprofil zu schärfen. Zweitens war Meinungsfreiheit ein Mittel, um Ansehen, Freiräume und Bezahlung einer gewachsenen Zahl von Parteiredakteuren zu verbessern. Diese konnten die Formierung von journalistischen Berufsinteressen in der Gesellschaft beobachten (vgl. Kutsch 2008).

\subsection{Das Problem der öffentlichen Darstellung}

Die Bewahrer mussten das Problem umdeuten, um nicht in Widerspruch zu den Grundsätzen der Partei zu geraten. Sie rückten die Außenwirkung der SPD in den Vordergrund.

Vorstandsmitglieder, prominente Parteiredakteure, Programmatiker oder lokale Funktionäre lehnten die Forderungen mit Hinweis auf die background ideational abilities der Partei ab. Stellvertretend sprach Ignaz Auer aus, was doch für alle Gewissheit sein musste, die in der Partei der Unterdrückten waren: Meinungsfreiheit ist „uns so sehr in Fleisch und Blut übergegangen“ (Protokoll 1891, S. 96). Damit 
schob der Vorstandssekretär die Kritik der linken Opposition beiseite. Später betonte auch Schatzmeister Wilhelm Pfannkuch, freie Kritik sei das, ,was unsere Partei auszeichnet“ (vgl. Protokoll 1895, S. 73). Der Vorsitzende Bebel beschwor Meinungsfreiheit als ,unser Lebensprinzip“, als es um Bernstein ging (Protokoll 1899, S. 127).

Dem Parteizentrum machte ein anderes Problem zu schaffen. Die öffentliche Kommunikation über innere Meinungsfreiheit schadete der Partei. Parteipresse und jegliche anderswo geübte öffentliche Kritik lieferten der bürgerlichen Presse Material, um die Sozialdemokratie zu bekämpfen. Der Parteipresse wurde vorgehalten, die offizielle Linie im Umgang mit Abweichlern zu ignorieren. Sie habe etwa zu zeigen, ,was die Masse der Parteigenossen über Bernstein denkt“ (Protokoll 1901, S. 157). Der Vorwurf, Kritik nicht intern vorgebracht zu haben, wurde im VorwärtsKonflikt laut (vgl. Büttner et al. 1905), in den Debatten um die Parteipresse und Theoriezeitschriften (vgl. Calwer 1901; Protokoll 1901, S. 189-190; Kautsky 1902, S. 807). Noch 1913 verurteilte der Parteiausschuss die Monatshefte für ihre ,hämische Art der Parteikritik, die nur den Gegnern dient, die Partei aber schädigt“ (SPD 1980, S. 58). Der Vorsitzende der Reichstagsfraktion Philipp Scheidemann behauptete im Widerspruch zu den Erfahrungen der Abweichler: ,Kein Parteigenosse braucht ein außerhalb der Partei stehendes Organ aufzusuchen, wenn er etwas zu sagen wünscht.“ (SPD 1980, S. 56).

Im Hintergrund operierte das moralische Presseverständnis. Die bürgerliche Presse habe die Debatten um Meinungsfreiheit ,natürlich (...) benützt, um der Sozialdemokratie sofort den Wunsch nach Unterdrückung der freien Meinungsäußerung anzudichten“, resümierte etwa der Parteitheoretiker Kautsky (1902, S. 807) den Parteitag 1902. Mehring war sich mit Kautsky einig, dass die ,kapitalistisch-liberale(n) Presse“ am stärksten wirke (Mehring 1909, S. 227; vgl. Kautsky 1893, S. 85). Den Bewahrern war klar, dass die revisionistischen Abweichler von der Sympathie eines Berliner Tageblatts profitieren konnten (vgl. Protokoll 1901, S. 159). Viele Arbeiterhaushalte bevorzugten das bürgerliche Lokalblatt gegenüber der Parteipresse (Protokoll 1910, S. 216). Die Parteiführung war sich auch der zunehmenden Bedeutung medialer Kommunikation bewusst. Auer verwies auf das Nachrichtenzentrum Berlin, als er die ,wenig angebrachte Art“ kritisierte (Protokoll 1891, S. 94), in der die linke Opposition in Berlin gegen „Diktatur und Unduldsamkeit“ in der Partei polemisiert hatte (Parteivorstand 1891, S. 20). Die Berliner „Preßorganisation“ trage ,alles sofort bis in den entferntesten Ort“" hinaus (vgl. Protokoll 1891, S. 95). Um der öffentlichen Aufmerksamkeit zu entgehen, versuchte die Parteiführung, zumindest ihre Presseprobleme nicht auf den Parteitagen, sondern auf einer nicht öffentlichen Konferenz zu erörtern (vgl. Protokoll 1896, S. 114).

Die diskursive Praxis der Bewahrer beinhaltete mehrere Strategien. Erstens untermauerten sie das Parteipresseverständnis mit den persönlichen Erfahrungen von Unterdrückung und Verfolgung der älteren Genossen, die ihre Distanz zur bürgerlichen Gesellschaft begründete (vgl. Protokoll 1897, S. 98). Zweitens stützten sie ihre Position, indem sie die Abweichler delegitimierten. Dabei wurde deren Loyalität infrage gestellt. Sie seien ,Schriftsteller (...), die sich zu uns gesellen, ohne die bürgerliche Denkweise völlig zu überwinden“ und ohne die Sympathie für ihre bürgerlichen Berufskollegen aufzugeben (Kautsky 1905, S. 225; vgl. Mehring 
1908). Den Sozialistischen Monatsheften, deren Auflage vor dem Ersten Weltkrieg selbst großzügig geschätzt mit 5000 Exemplaren nur halb so hoch war wie die der Neuen Zeit (vgl. Drahn 1930, S. 400), unterstellte Kautsky außerdem „,kapitalistische Methoden“ (Kautsky 1902, S. 809). Mehring behauptete sogar, die bürgerliche Presse legitimiere mit der Idee der Meinungsfreiheit lediglich kapitalistische Interessen (vgl. Mehring 1908, S. 4). Die Vorwärts-Redakteure um Eisner sahen sich schließlich dem Vorwurf ausgesetzt, ihre Verantwortung missbraucht und die Partei verraten zu haben (vgl. Büttner et al. 1905, S. 139). Von der „Moralrichterei“ und „Pharisäerei“ Kautskys nach der Jahrhundertwende hatte selbst Freund Victor Adler genug, der auch Bebel „Unerbittlichkeit“ in Sachen Mitarbeit in der bürgerlichen Presse vorwarf (Adler 1954, S. 427, S. 421).

Das Problem der Außenwirkung mündete in das Gebot der disziplinierten Parteiöffentlichkeit. Mit der Änderung des Organisationsstatuts versuchte die Parteiführung nach Ende des Sozialistengesetzes, die Parteipresse einzuhegen (vgl. Protokoll 1890, S. 154). Die Führung konnte sich dabei auf eine überwältigende Parteimehrheit stützen (vgl. Protokoll 1890, S. 1247-1248). Einfache Mitglieder, wie der Thüringer Delegierte Grunwald, betonten in der Bernstein-Debatte, zur Kritik seien ,nur Parteivereine da“, und wollten die Orte des Sprechens einschränken (Protokoll 1901, S. 156). Auch die lokalen Verbände strebten mit der Einrichtung von Pressekommissionen die Kontrolle der Presse durch die Partei an (vgl. Protokoll 1891, S. 230). Neben der breiten Zustimmung der Basis schienen auch die Wahlerfolge den Kurs zu bestätigen. Kontinuierlich warnte die Parteiführung vor privaten Neugründungen wegen des wirtschaftlichen Risikos (vgl. Protokoll 1877, S. 25, 1880, S. 49, 1892, S. 40-41, 1894, S. 68-69, 1897, S. 98-99, 1902, S. 162-163, 1912, S. 208). Dieser Argumentation folgte bislang auch die Literatur (vgl. Danker et al. 2003, S. 15; Koszyk 1980, S. 13-14). Doch aus Medialisierungssicht verbirgt sich darin auch der Wunsch nach Einhegung und Kontrolle in einem sich ausdifferenzierenden Mediensystem. Erstens las ,ein großer Teil“ der Wähler ,,bürgerliche Blätter“ (Protokoll 1913, S. 251), und die Parteipresse drang nicht ,in die indifferenten Massen“ (Protokoll 1913, S. 244) vor. Zweitens befürchtete die Parteileitung, dass weitere sozialdemokratische Titel das erwünschte einheitliche Bild der Partei gefährdeten. Scheidemann monierte etwa, ,daß manche unserer Blätter fast ausschließlich für die eigenen Parteigenossen schreiben und dabei vergessen, daß das Parteiorgan das wichtigste Werbemittel der Partei sein soll“" (Protokoll 1913, S. 226).

Die Position der Bewahrer lässt sich einerseits durch die materielle Versorgung besoldeter Funktionäre wie Pfannkuch und Auer oder wichtiger Parteiredakteure wie Kautsky und Mehring erklären. Für den finanziell unabhängigen Bebel trifft dieses Argument nicht zu (vgl. Schmidt 2013, S. 166-168). Andererseits dürfte ihre Rolle, etwa im Vorstand, von ihnen verlangt haben, Probleme so darzustellen, dass Geschlossenheit sowie Sichtbarkeit für bestehende Macht- und Mehrheitsverhältnisse mit der Basis gesichert wurden.

Die Bewahrer konnten Diskursforen und -regeln wesentlich bestimmen und die Parteimehrheit mobilisieren. Das stabilisierte den Zwiespalt zwischen Parteipresseverständnis und Meinungsfreiheit. 
Die Bewahrer setzten sich in den Auseinandersetzungen durch. Wir machen ihren Erfolg an den Nachwirkungen fest, die der Diskurs für die Abweichler hatte: Parteitagsbeschlüsse, Parteiausschlüsse oder Parteiaustritte, Entlassungen oder Kündigungen und der Verlust des politischen Mandats. Parteiausschlüsse oder Ausschlussverfahren trafen die linken Oppositionellen 1891 wie zuvor Johann Most und Wilhelm Hasselmann (vgl. Wienand 1976, S. 208). Die Vorwärts-Redakteure kamen ihrer Entlassung durch Kündigung zuvor (vgl. Grau 2001, S. 217). Sowohl der Beschluss zum Verbot der Mitarbeit an bürgerlichen Zeitungen kam mit überwältigender Mehrheit zustande (vgl. Sperlich 1983, S. 112), als auch der, Parteischädigung (verstanden als „grobe Verstöße gegen die Parteidisziplin“, Protokoll 1909, S. 382-383) als Grund für Parteiausschluss zu deklarieren. Die Mehrheit konnte für das moralische Presse- und Öffentlichkeitsverständnis mobilisiert werden. Paul Göhre legte nach der Kontroverse mit Bebel um die Mitarbeit in bürgerlichen Zeitungen sein Reichstagsmandat nieder, das er ein paar Jahre später neu gewann (vgl. Bloch 2008, S. 11-12). Auf dem Parteitag gelobte er, nicht mehr für die Zukunft zu schreiben und trat aus der Kirche aus (vgl. Protokoll 1903, S. 238). Auch Georg Bernhard leistete dort öffentlich Abbitte. Heinrich Braun verlor nach diesem Parteitag sein Mandat (vgl. Braun-Vogelstein 1932, S. 292). Bebel stand auf der Tribüne und „hypnotisierte“ den Parteitag, erinnerte sich Stampfer (1957, S. 90). Das häufig beschriebene Charisma des Parteiführers, seine Inszenierungen, aber auch der Personenkult um den „Kaiser der Arbeiter" haben Abstimmungen sicherlich mit beeinflusst (vgl. Schmidt 2013, S. 229). Während Richard Calwers Parteiaustritt, angeblich Folge ,gezielter Angriffe auf seine persönliche Integrität", offenbar keine größeren Wellen schlug (vgl. Bloch 2008, S. 16, 20), waren das „Stück Macht u. Prestige“, das der Partei mit einem Austritt von Georg von Vollmar und Wolfgang Heine ,verloren gehen“" würde, zumindest hinter den Kulissen ein Thema (Adler 1954, S. 422).

Unserer theoretischen Perspektive folgend, kann der Umgang mit den Abweichlern mit der Kontrolle erklärt werden, die die Bewahrer über öffentliche Parteiforen ausübten (Macht über Ideen). Auch wenn die Herausforderer nach 1890 auf den Parteitagen präsent waren, Heinrich Braun seine Sicht auf die Mitarbeit in bürgerlichen Zeitungen im Vorwärts veröffentlichen durfte und die Monatshefte zur publizistischen Heimat prominenter Parlamentarier wurden, so dass Mittmann (1976, S. 237) diesen sogar einen ,halboffiziellen Status“ zugeschrieben hat: Die Bewahrer im Parteizentrum bestimmten den Zugang zu den Foren, in denen die herrschenden Ideen der Partei deklariert wurden. Als Vorstandsmitglieder und lokale Funktionäre in Pressekommissionen oder als mehrheitstreue Parteiredakteure beeinflussten sie, wer in den offiziell anerkannten Parteiblättern im Machtzentrum schreiben durfte, welche Redakteure ausgewechselt werden sollten und was auf die Tagesordnung der Parteitage kommen sollte. Karl Kautsky (1960, S. 542) zumindest empfand rückblickend, dass er nach dem Ausscheiden von Heinrich Braun aus der Redaktion der Neuen Zeit „,von niemand mehr beschränkt" wurde.

Die Disziplinierung der Kritiker ist aber nicht ohne die institutionellen Kontexte der Organisation zu verstehen. Die Erfahrung der Ausgrenzung hielt an, obwohl die Partei zunehmend in den Staat hineinwuchs. Politik und Industrie machten Öffentlichkeitsarbeit gegen die Sozialdemokratie, während sich die Strukturen der Nachrichtenorganisation bis zum Vorabend des Ersten Weltkriegs weiter verdichteten (vgl. 
Wilke 2008, S. 259-260; Kunczik 1997, S. 192). Disziplinierende Elemente wurden dadurch stabilisiert, die Idee der Anzeigenunabhängigkeit war aufgegeben worden (vgl. Löblich und Venema 2018). Auch die einmal geschaffenen Einrichtungen der Parteipresse dürften die Autorität des ihnen zugrundeliegenden Presseverständnisses weiter gestützt haben (vgl. Carstensen und Schmidt 2016, S. 329). Die Entwicklung zur Massenpartei und die Wahlerfolge gaben dem marxistischen Zentrum Rückhalt, das sich aber von der Idee, Revolution zu machen, bis zum Ersten Weltkrieg verabschiedet hatte (vgl. Faulenbach 2012, S. 31).

Auch wenn bis 1914 die Parteigenossen auf eine disziplinierte Parteiöffentlichkeit eingeschworen wurden, blieb der Zwiespalt bestehen, in den die SPD nach dem ersten Medialisierungsschub geraten war. Innere Meinungsfreiheit war weiterhin an Bedingungen gebunden, die Presse der Kontrolle der Funktionäre unterstellt. Die Sekundärliteratur liefert Anhaltspunkte für das Anhalten des Zwiespalts. Ein paar Jahre später waren ehemalige Parteiführer an den Rand gedrängt. Die offizielle Parteilinie hatte sich zu den Reformisten verschoben. Karl Kautsky, Clara Zetkin und ihre Anhänger waren nun eine Minderheit in der Partei. Als Kriegsgegner waren sie keine „Gatekeeper“ mehr und konnten diese Meinung nicht ungehindert äußern (vgl. Koszyk 1958, S. 59; Löblich und Venema 2019). Doch setzte das gewandelte Parteizentrum seine Kritik an der Mitarbeit in bürgerlichen Blättern fort (vgl. Protokoll 1917, S. 32). Die Kritik an fehlender Meinungsfreiheit kehrte zurück. Als das Radio längst eingeführt war, verabschiedete der Parteitag verschärfte Regeln für öffentliche Kommunikation, und journalistische „Eigenbrötelei“ wurde mit Ausschluss bedroht. Wieder blieben der Opposition nur eigene Organe (vgl. Koszyk 1958, S. 192).

\section{Fazit}

Mit der expandierenden Medienwelt der 1890er Jahre geriet die SPD in einen Zwiespalt. Die Kritik an mangelnder innerer Meinungsfreiheit verstärkte sich. Abweichler von der Parteilinie forderten Meinungsfreiheit aus dem Wunsch heraus, Sichtbarkeit und Legitimation für ihre politischen Ideen zu bekommen und den Kurs der Partei zu ändern. Parteijournalisten formulierten ihre Kritik, um über die Anerkennung ihres Berufsstands eine bessere Ausstattung der Redaktionen zu erwirken. Die Partei hatte einerseits stärker als in den Jahren zuvor Meinungsfreiheit auch auf sich selbst anzuwenden. Andererseits musste sie einem einheitlichen Bild in der Öffentlichkeit mehr Bedeutung beimessen.

Der öffentlich ausgetragene Parteidiskurs kann aus Medialisierungsperspektive als Reaktion der Organisation(-smehrheit) auf die wachsende Bedeutung öffentlicher Kommunikation verstanden werden. Sogar Kritiker blieben teilweise dem Parteipresseverständnis verhaftet. Die Bekämpfung der Kritiker konnte den Zwiespalt aber nicht auflösen, denn sie änderte nichts an der strukturellen Verankerung medialer Aufmerksamkeit und öffentlicher Legitimation. Es gibt Anhaltspunkte für die Annahme, dass dieser Zwiespalt die SPD auch während der folgenden Medialisierungsschübe im 20. Jahrhundert beschäftigt hat und außerdem in der Geschichte anderer Akteure mit Außenseitererfahrung (Gewerkschaften, politischer Arm des Katholizismus) zu finden ist. Der vorliegende Beitrag stützte sich auf eine Verbin- 
dung von (organisationsbezogener) Medialisierungsforschung und diskursivem Institutionalismus. Diese Kombination dürfte auch jenseits unseres Erkenntnisinteresses sinnvoll sein. Schmidts Analysewerkzeuge lieferten hier einen Blick in das Innere einer Organisation: wie historisch gewachsene Gewissheiten den Diskurs strukturiert haben, aber auch, wie im Diskurs Institutionen infrage gestellt und Veränderungen angestoßen wurden. Sie lenken den Blick außerdem auf Außenseiter und Akteure im Zentrum, auf Abweichung und Sanktionierung. Medialisierung trug zur Erklärung bei: Der Diskurs um Meinungsfreiheit in der Partei wurde als Folge eines Medienwandels eingeordnet, der auch Machtfragen aufwarf. Die Integration von Diskurs und Ideen in die (hier angesprochene) Medialisierungsliteratur macht der Forschung einen Aspekt zugänglich: die teils widersprüchlichen, jedenfalls nicht determinierten und auch nicht immer nur strategischen Reaktionen auf die gewachsene Bedeutung öffentlicher Kommunikation (vgl. Donges und Jarren 2014, S. 188).

Ohne Anschluss an eine Forschungsrichtung wie Medialisierung fehlt dem politikwissenschaftlichen Ansatz das Wissen um gesellschaftliche Kommunikation. Ihm fehlen Erkenntnisinteressen der Kommunikationswissenschaft. Auch bleibt die Verbindung von Akteuren und Diskurs mit der gesellschaftstheoretischen Ebene im diskursiven Institutionalismus unterbelichtet. Diese Verbindung ist für kommunikationsgeschichtliche Studien wichtig, die Ereignisse in ihrem Kontext verstehen wollen. Medialisierungsansätze können einen gesellschafts- und medientheoretischen Gesamtzusammenhang herstellen.

Die Diskursmerkmale, die wir für die SPD nach dem ersten Medialisierungsschub im Kaiserreich ermittelt haben, können mit derselben Theorieperspektive langfristig auf Kontinuitäten und Veränderungen untersucht werden. Ein aktuelles Forschungsbeispiel dürfte Kevin Kühnert im letzten Jahr geliefert haben. Der Juso-Chef hatte Kapitalismuskritik geäußert und damit nicht nur Unruhe in den Leitmedien ausgelöst, sondern auch Kritik aus der Parteispitze (vgl. o.V. 2019). Zu untersuchen wäre, ob und wie auch unter veränderten institutionellen Bedingungen (etwa Staatsform, Partei- und Mitgliederstrukturen) parteiinterne Machtkämpfe um Meinungsfreiheit als Medialisierungsfolge verstanden werden können und welche Auswirkungen diese Kämpfe heute haben.

Förderung Die Studie wurde nicht mit externen oder hochschulinternen finanziellen Mitteln gefördert und ist nicht im Rahmen von Lehrveranstaltungen entstanden.

Funding Open Access funding provided by Projekt DEAL.

Open Access Dieser Artikel wird unter der Creative Commons Namensnennung 4.0 International Lizenz veröffentlicht, welche die Nutzung, Vervielfältigung, Bearbeitung, Verbreitung und Wiedergabe in jeglichem Medium und Format erlaubt, sofern Sie den/die ursprünglichen Autor(en) und die Quelle ordnungsgemäß nennen, einen Link zur Creative Commons Lizenz beifügen und angeben, ob Änderungen vorgenommen wurden.

Die in diesem Artikel enthaltenen Bilder und sonstiges Drittmaterial unterliegen ebenfalls der genannten Creative Commons Lizenz, sofern sich aus der Abbildungslegende nichts anderes ergibt. Sofern das betreffende Material nicht unter der genannten Creative Commons Lizenz steht und die betreffende Handlung nicht nach gesetzlichen Vorschriften erlaubt ist, ist für die oben aufgeführten Weiterverwendungen des Materials die Einwilligung des jeweiligen Rechteinhabers einzuholen.

Weitere Details zur Lizenz entnehmen Sie bitte der Lizenzinformation auf http://creativecommons.org/ licenses/by/4.0/deed.de. 


\section{Literatur}

Adler, V. (1954). Briefwechsel mit August Bebel und Karl Kautsky, sowie Briefe von und an Ignaz Auer, Eduard Bernstein, Adolf Braun, Heinrich Dietz, Friedrich Ebert, Wilhelm Liebknecht, Hermann Müller und Paul Singer, gesammelt und erläutert von Friedrich Adler. Wien: Verlag der Wiener Volksbuchhandlung.

Bebel, A. (1903). Erklärung. Die Neue Zeit, 21(41), 449.

Bernstein, E. (1895). Aus Anlaß eines Sensationsprozesses. Die Neue Zeit, 32(13), 171-176.

Bernstein, E. (1896). Aus früheren Kämpfen. Allerlei aus den Erinnerungen eines englischen Agitators. Die Neue Zeit, 14(27), 11-18.

Bernstein, E. (1909). Das Recht des sozialdemokratischen Schriftstellers. Sozialistische Monatshefte, 13, 1090-1095.

Bernstein, E. (1991). Sozialdemokratische Lehrjahre: Entwicklungsgang eines Sozialisten. Berlin: Dietz Verlag.

Bezirksleitung Berlin der SED (1987). Geschichte der revolutionären Berliner Arbeiterbewegung. Bd. 1. Berlin: Dietz Verlag.

Bloch, M. (2008). Die Sozialistischen Monatshefte und die Akademikerdebatte in der deutschen Sozialdemokratie vor 1914: Die „Fälle“ Göhre, Schippel, Calwer und Hildebrand. Mitteilungsblatt des Instituts für soziale Bewegungen, 40, 7-22.

Boll, F. (2002). Die deutsche Sozialdemokratie und ihre Medien. Wirtschaftliche Dynamik und rechtliche Formen. Bonn: J.H.W. Dietz.

Bösch, F. (2004). „Berlusconi von links“. Die Medien der SPD in historischer Perspektive. Archiv für Sozialgeschichte, 44, 501-508.

Bösch, F., \& Frei, N. (2006). Die Ambivalenz der Medialisierung. Eine Einführung. In F. Bösch \& N. Frei (Hrsg.), Medialisierung und Demokratie im 20. Jahrhundert (S. 7-23). Göttingen: Wallstein Verlag.

Braun, H. (1893). Zur Lage der deutschen Sozialdemokratie. Archiv für soziale Gesetzgebung und Statistik, $6,506-520$.

Braun, H. (1903a, 29. März). Freiheit der Meinungsäußerung und Parteivorstand. Vorwärts, S. 3-4.

Braun, H. (1903b). Zur Frage der Freiheit und des Verhaltens der Leipziger Volkszeitung. Berlin: Selbstverlag.

Braun-Vogelstein, J. (1932). Ein Menschenleben. Heinrich Braun und sein Schicksal. Tübingen: Rainer Wunderlich.

Brenning, J. (1980). Christentum und Sozialdemokratie. Paul Göhre, Fabrikarbeiter, Pfarrer, Sozialdemokrat. Eine Sozialethisch-historische Untersuchung. Diss. Marburg: Universität Marburg.

Büttner, P., Gradnauer, G., Eisner, K., Kaliski, J., Schröder, W., \& Wetzker, H. (Hrsg.). (1905). Der Vorwärts-Konflikt. Gesammelte Aktenstücke. München: Kommissions-Verlag G. Birk \& Co.

Calwer, R. (1894). Das Kommunistische Manifest und Die heutige Sozialdemokratie. Braunschweig: A. Günther.

Calwer, R. (1901). Die socialdemokratische Presse. Sozialistische Monatshefte, 5, 699-704.

Calwer, R. (1906). Disziplin und Meinungsfreiheit. Sozialistische Monatshefte, 10, 36-40.

Carstensen, M., \& Schmidt, V. (2016). Power through, over and in ideas: conceptualizing ideational power in discursive institutionalism. Journal of European Public Policy, 23, 318-337.

Danker, U., Oddey, M., Roth, D., \& Schwabe, A. (2003). Am Anfang standen Arbeitergroschen. 140 Jahre Medienunternehmen der SPD. Bonn: J.H.W. Dietz.

Donges, P. (2008). Medialisierung politischer Organisationen. Parteien in der Mediengesellschaft. Wiesbaden: VS.

Donges, P., \& Jarren, O. (2014). Mediatization of political organizations: changing parties and interest groups? In F. Esser \& J. Strömbäck (Hrsg.), Mediatization of politics: understanding the transformation of western democracies (S. 181-199). Basingstoke: Palgrave Macmillan.

Donges, P., \& Nitschke, P. (2018). Political organizations and their online communication. Sociology Compass, 12(2), 1-10.

Drahn, E. (1930). Zur Quellenkunde einer Pressegeschichte der Sozialisten (Marxisten) Deutschlands. Jahrbücher für Nationalökonomie und Statistik, 81(132), 393-409.

Faulenbach, B. (2012). Geschichte der SPD: Von den Anfängen bis zur Gegenwart. München: C.H. Beck.

Fricke, D. (1987). Handbuch zur Geschichte der deutschen Arbeiterbewegung 1869 bis 1917. Bd. 1. Berlin: Dietz.

Geppert, D. (2007). Pressekriege. Öffentlichkeit und Diplomatie in den deutsch-britischen Beziehungen (1896-1912). München: Oldenbourg. 
Göhre, P. (1891). Drei Monate Fabrikarbeiter und Handwerksbursche. Eine praktische Studie. Leipzig: Fr. Wilh. Grunow.

Grau, B. (2001). Kurt Eisner. 1867-1919. Eine Biographie. München: Beck.

Große Kracht, K. (2010). Presse und Kanzel. Päpstliches Medienverständnis und katholische Publizistik in Deutschland (1920-1970er Jahre). In U. Daniel \& A. Schildt (Hrsg.), Massenmedien im Europa des 20. Jahrhunderts (S. 331-356). Köln: Böhlau.

Groth, O. (1929). Die Zeitung. Ein System der Zeitungskunde (Journalistik). Bd. 2. Mannheim: J. Bensheimer.

Günther, K. (1973). Die andere Meinung in der SPD 1949, 1955/56, 1958/61. Ein Beitrag zum Problem innerparteilicher Diskussionsfreiheit. Archiv für Sozialgeschichte, 8, 24-52.

Hall, A. (1977). Scandal, Sensation and Social Democracy. The SPD Press and Wilhelmine Germany 1890-1914. Cambridge: Cambridge University Press.

Heine, W. (1901). Notwendige Reformen im Pressrecht. Sozialistische Monatshefte, 5, 243-252.

Heine, W. (1903). Der 16. Juni. Sozialistische Monatshefte, 7, 475-478.

Kampffmeyer, P. (1930). Georg von Vollmar. München: Birk.

Kautsky, K. (1893). Der Parlamentarismus, die Volksgesetzgebung und die Sozialdemokratie. Stuttgart: J. H. W. Dietz..

Kautsky, K. (1902). Der Münchener Parteitag. Die Neue Zeit, 20(52), 804-809.

Kautsky, K. (1905). Der Journalismus in der Sozialdemokratie. Die Neue Zeit, 24(7), 216-225.

Kautsky, K. (1960). Erinnerungen und Erörterungen. Mouton: 's-Gravenhage.

Kohlrausch, M. (2005). Der Monarch im Skandal. Die Logik der Massenmedien und die Transformation der wilhelminischen Monarchie. Berlin: Akademie Verlag.

Koszyk, K. (1958). Zwischen Kaiserreich und Diktatur. Die sozialdemokratische Presse von 1914 bis 1933. Heidelberg: Quelle \& Meyer.

Koszyk, K. (1980). Die Geschichte der sozialdemokratischen Presse im Überblick. In K. Koszyk \& G. Eisfeld (Hrsg.), Die Presse der deutschen Sozialdemokratie (S. 3-58). Bonn: Verlag Neue Gesellschaft.

Koszyk, K. (1989). Die Arbeiterpresse: Organisation, Probleme, Wirkung. Ein historischer Überblick. $G e-$ werkschaftliche Monatshefte, 40(3), 172-184.

Kunczik, M. (1997). Geschichte der Öffentlichkeitsarbeit in Deutschland. Köln: Böhlau.

Kutsch, A. (2008). Journalismus als Profession. Überlegungen zum Beginn des journalistischen Professionalisierungsprozesses in Deutschland am Anfang des 20. Jahrhunderts. In A. Blome \& H. Böning (Hrsg.), Presse und Geschichte. Leistungen und Perspektiven der historischen Presseforschung (S. 289-325). Bremen: edition lumière.

Leuthner, K. (1910). Wandlungen der Journalistik. Sozialistische Monatshefte, 14, 488-496.

Löblich, M., \& Venema, N. (2018). Sozialdemokratie und Medienpolitik. Der Beitrag parteiungebundener Autoren in der Pressereformdebatte Anfang des 20. Jahrhunderts. Medien und Kommunikationswissenschaft, 66, 320-336.

Löblich, M., \& Venema, N. (2019). Die SPD und ihre Frauenpresse. Die Gleichheit im Parteidiskurs nach Ausbreitung der Massenpresse. Medien \& Zeit, 34(4), 29-41.

Loreck, J. (1977). Wie man früher Sozialdemokrat wurde. Das Kommunikationsverhalten in der deutschen Arbeiterbewegung und die Konzeption der sozialistischen Parteipublizistik durch August Bebel. Bonn-Bad Godesberg: Neue Gesellschaft.

Matthias, E., \& Miller, S. (1966). Das Kriegstagebuch des Reichstagsabgeordneten Eduard David 1914 bis 1918. Düsseldorf: Droste.

Mehring, F. (1908). Bürgerliche und proletarische Presse. Die Neue Zeit, 26(27), 1-4.

Mehring, F. (1909). Die öffentliche Meinung. Die Neue Zeit, 28(7), 225-228.

Meißner, M. (2017). Der Verein Arbeiterpresse (1900-1933). Selbstverständnis, Autonomie und Ausbildung sozialdemokratischer Redakteure. Baden-Baden: Nomos.

Meyen, M. (2009). Medialisierung. Medien Kommunikationswissenschaft, 57, 23-38.

Meyen, M., Thieroff, M., \& Strenger, S. (2014). Mass media logic and the mediatization of politics: a theoretical framework. Journalism studies, 15(3), 271-288.

Mittmann, U. (1976). Fraktion und Partei. Ein Vergleich von Zentrum und Sozialdemokratie im Kaiserreich. Düsseldorf: Droste.

Morina, C. (2017). Die Erfindung des Marxismus. Wie eine Idee die Welt eroberte. München: Siedler.

Ohne Verfasser (2019, 3. Mai). Nahles findet Kühnerts Thesen falsch. Der Spiegel. https://www.spiegel.de/ politik/deutschland/kevin-kuehnert-andrea-nahles-nennt-thesen-des-juso-chefs-falsch-a-1265654.

html. Zugegriffen: 9. März 2020

Parteivorstand (1903a, 3. März). Partei-Nachrichten. Vorwärts, S. 3.

Parteivorstand (1903b, 31. März). Partei-Nachrichten. Vorwärts, S. 5. 
Parteivorstand (1891). Die Anschuldigungen der Berliner Opposition. Berlin: Verlag der Expedition des „Vorwärts“, Berliner Volksblatt.

Plener, U. (1961). Karl Kautskys Opportunismus in Organisationsfragen (1900 bis 1914). Zur Entstehung des Zentrismus in der deutschen Sozialdemokratie. Beiträge zur Geschichte der deutschen Arbeiterbewegung, 3, 349-470.

Protokoll des Kongresses der Deutschen Sozialdemokratie, abgehalten auf Schloß Wyden in der Schweiz am 20. bis 23. August 1880. Zürich: Verlag von A. Herter

Protokoll des Socialisten-Congresses zu Gotha, vom 19. bis 23. August 1876. Berlin: Druck und Verlag der Allgemeinen Deutschen Associations-Buchdruckerei.

Protokoll des Vereinigungs-Congresses der Sozialdemokraten Deutschlands abgehalten zu Gotha, vom 22. bis 27. Mai 1875. Leipzig: Druck und Verlag der Genossenschaftsbuchdruckerei.

Protokolle über die Verhandlungen der Parteitage der Sozialdemokratischen Partei Deutschlands (1890-1959). Online-Edition der Bibliothek der Friedrich-Ebert-Stiftung. http://library.fes.de/parteitage. Zugegriffen: 06. Sept. 2019.

Requate, J. (1995). Journalismus als Beruf. Entstehung und Entwicklung des Journalistenberufs im 19. Jahrhundert. Deutschland im internationalen Vergleich. Göttingen: Vandenhoeck \& Ruprecht.

Requate, J. (2006). Kommerzialisierung der Presse im frühen 20. Jahrhundert. Konsumierendes und fragmentiertes Publikum. In C. Zimmermann (Hrsg.), Politischer Journalismus, Öffentlichkeiten und Medien im 19. und 20. Jahrhundert (S. 121-137). Ostfildern: Thorbecke.

Ressmann, W. (1991). Strukturprobleme sozialdemokratischer Medienunternehmen: Eine organisationspolitische Analyse der SPD-Presseunternehmen von den Anfängen bis zur Gegenwart. Wiesbaden: Deutscher Universitätsverlag.

Ritter, G. A. (1963). Die Arbeiterbewegung im Wilhelminischen Reich (2. Aufl.). Berlin: Colloquium Verlag.

Rudolph, K., Bitzegeio, U., Fattmann, R., \& Scholten, J. (2003). Die unternehmerische Tätigkeit der Arbeiterbewegung als neues Forschungsfeld. Moving the Social, 28, 275-294.

Schmidt, J. (2013). August Bebel. Kaiser der Arbeiter. Zürich: Rotpunktverlag.

Schmidt, V.A. (2008). Discursive institutionalism: the explanatory power of ideas and discourse. Annual Review of Political Science, 11, 303-326.

Schröder, W. (1907). Unsere Presse. Sozialistische Monatshefte, 11, 741-749.

Schröder, W. (1910). Handbuch der sozialdemokratischen Parteitage von 1863 bis 1909. München: Birk.

Sozialdemokratische Partei Deutschlands (1980). Protokolle der Sitzungen des Parteiausschusses der SPD 1912 bis 1921. Nachdruck. Bd. 1. Berlin: J.H.W. Dietz.

Sperlich, W. (1983). Journalist mit Mandat. Sozialdemokratische Reichstagsabgeordnete und ihre Arbeit in der Parteipresse 1867 bis 1918. Düsseldorf: Droste.

Stampfer, F. (1903). Gewissensfragen. Die Neue Zeit, 21(26), 825-828.

Stampfer, F. (1957). Erfahrungen und Erkenntnisse. Aufzeichnungen aus meinem Leben. Köln: Verlag für Politik und Wirtschaft.

Steinbach, P. (1990). Die Entwicklung der deutschen Sozialdemokratie im Kaiserreich im Spiegel der historischen Wahlforschung. In G. A. Ritter (Hrsg.), Der Aufstieg der deutschen Arbeiterbewegung: Sozialdemokratie und Freie Gewerkschaften im Parteiensystem und Sozialmilieu des Kaiserreichs (S. 1-35). München: Oldenbourg.

Stöber, G. (2000). Pressepolitik als Notwendigkeit. Zum Verhältnis von Staat und Öffentlichkeit im Wilhelminischen Deutschland 1890-1914. Stuttgart: Steiner.

Stöber, R. (2014). Deutsche Pressegeschichte. Von den Anfängen bis zur Gegenwart (3. Aufl.). Konstanz: UVK.

Th. (1906). Meinungsfabriken. Mitteilungen des Vereins Arbeiterpresse, 7(55), 1-2.

von Vollmar, G. (1892). Ueber Staatssocialismus. Nürnberg: Wörlein.

von Vollmar, G. (1903). Lehren und Folgen der letzten Reichstagswahlen. München: G. Birk \& Co.

Wehler, H.-U. (2008). Deutsche Gesellschaftsgeschichte. Von der „Deutschen Doppelrevolution“ bis zum Beginn des Ersten Weltkrieges: 1849-1914. Bd. 3. München: Beck.

Wetzel, H.-W. (1975). Presseinnenpolitik im Bismarckreich (1874-1890). Das Problem der Repression oppositioneller Zeitungen. Frankfurt am Main: Peter Lang.

Wienand, P. (1976). Revoluzzer und Revisionisten. Die „Jungen“ in der Sozialdemokratie vor der Jahrhundertwende. Politische Vierteljahresschrift, 17, 208-241.

Wilke, J. (2008). Grundzüge der Medien- und Kommunikationsgeschichte (2. Aufl.). Köln: Böhlau.

Dr. Maria Löblich ist Professorin für Publizistik- und Kommunikationswissenschaft an der FU Berlin. 
Niklas Venema ist wissenschaftlicher Mitarbeiter am Institut für Publizistik- und Kommunikationswissenschaft der FU Berlin. 